\title{
Therapeutic options for Corona Virus Disease 2019 (COVID-19)
}

\begin{abstract}
The aim of this review article is to put forth the therapeutic options for coronavirus disease 2019 (COVID-19). There is no specific treatment or therapy recommended for COVID-19 up to now. The therapeutic approaches should be ordered according to the severity of disease. These options can be listed from one to ten such as isolation; oxygen support; respiratory treatment; anticoagulant treatment; anti-inflammatory drugs; hydroxychloroquine and combinations therapies; antiviral drugs; convalescent plasma therapy; mesenchymal stem cells therapy and vaccination. According to the severity and stage of the disease, suitable options are recommended.
\end{abstract}

Keywords: Coronavirus, treatment, therapeutic options, COVID-19
Volume I3 Issue 4 - 202 I

\author{
Ahmet Eroglu,' Seyfi Kartal,' Esra Kongur ${ }^{3}$ \\ 'Department of Anesthesiology and Intensive Care Medicine, \\ Karadeniz Technical University, Turkey \\ 2Department of Anesthesiology and Reanimation, Kanuni \\ Training and Research Hospital, University of Health Science, \\ Turkey \\ ${ }^{3}$ Department of Anesthesiology and Reanimation, Kanuni \\ Training and Research Hospital, Turkey
}

\author{
Correspondence: Ahmet Eroglu, Department of \\ Anesthesiology and Intensive Care Medicine, Karadeniz \\ Technical University, Trabzon, Turkey, \\ Email erogluah@hotmail.com
}

Received: June 13, 202। | Published: June 22, 202 |

\section{Introduction}

The first cases of coronavirus disease 2019 (COVID-19) were reported from Wuhan, China in December 2019, and then the disease rapidly spread to all of the world. The World Health Organization officially declared that it is a global pandemic on March 11, 2020. As of June 11, 2021, the World health Organization has reported almost 175, 929, 833 confirmed cases and 3797,918 deaths of COVID-19 globally. The clinical spectrum of COVID-19 varies from asymptomatic from to requiring mechanical ventilation and advanced support in the Intensive Care Unit. The common symptoms of disease are fever, dry cough, shortness of breath, dyspnea, poor appetite, myalgia or fatigue, sore throat, headache, chest pain, loss of taste or smell and diarrhea. ${ }^{1}$ The symptoms of COVID-19 can range from very mild to severe according to the disease severity. COVID-19 can cause pneumonia, severe acute respiratory syndrome coronavirus 2 (SARS-CoV-2), cardiac problems, coagulopathies, acute kidney injury, cytokine storm syndrome and multiple organ failures. The Angiotensin I Converting Enzyme 2 (ACE2) may play a role of pathogenesis of coronavirus. The $\mathrm{S}$ protein on coronavirus surface specially recognizes the spike protein in the ACE2 of exposed cell and after binding, the virus enters the cell thereby infecting it. Absolutely, the ACE2 receptor is widely disturbed on human cell surface, especially the lungs, heart, liver, kidneys and digestive organs. This can explain why beyond lung failure, the patients with COVID-19 may also exposure to acute myocardial injury, arrhythmia, myocarditis, acute kidney injury, coagulopathy, shock, and death from multiple organ failures. ${ }^{1}$

Up to now, there is no specific treatment or therapy strongly recommended for coronavirus disease 2019 (COVID-19). The therapeutic approaches should be ordered according to the severity and stage of the disease. The aim of this review article is to research the therapeutic options of COVID- 19 .

\section{Methods}

Publications on treatments and therapeutic approaches related to corona virus disease 2019 were searched in the English literature between 2019 and 2021. The review of the publications was done by a clinical team that has been involved in the treatment of COVID-19 patients from the beginning. This team's own experience also played a role in the choice and evaluation of the publications.

\section{Results}

\section{The therapeutic options for coronavirus disease 2019 (COVID-19)}

These options and suggestions can be listed from one to ten (Table 1):

Table I Therapeutic options and suggestions for COVID-19

\begin{tabular}{ll}
\hline Therapeutic options & Suggestions \\
\hline Isolation & rest, fluid and food intake \\
Oxygen support & $\begin{array}{l}\text { oxygen mask or nasal cannula } \\
\text { High-flow nasal cannula (HFNC), } \\
\text { continuous positive airway pressure } \\
\text { (CPAP), noninvasive ventilation (NIV) or } \\
\text { invasive mechanical ventilation (MV) }\end{array}$ \\
$\begin{array}{ll}\text { Respiratory treatment } & \text { Enoxaparin, Heparin } \\
\text { Anticoagulant treatment } & \text { Steroids, Tocizilumab, Anakinra, Ruxolitinib } \\
\text { Hydroxychloroquine and } & \text { Hydroxychloroquine alone or plus azith- } \\
\text { combinations therapies } & \text { romycin } \\
\text { Antiviral drugs } & \text { Remdesivir, Favipiravir, Lopinavir/Ritonavir } \\
\text { Convalescent Plasma } & \text { potential } \\
\text { Therapy } & \\
\text { Mesenchymal Stem Cells } \\
\text { Therapy }\end{array}$ & $\begin{array}{l}\text { one million cells/body weight iv } \\
\text { Vaccination }\end{array}$ \\
\hline
\end{tabular}

\section{Isolation, rest, fluid and food intake}

If a person has mild symptoms or none but real-time PCR assay is positive isolation, rest, and fluid and food intake should be recommended. 


\section{Oxygen support}

The aim of the oxygen support is to increase in fractional of inspiration oxygen (FiO2) in the patients with COVID-19. If the peripheral oxygen saturation $(\mathrm{SpO} 2)<90 \%$ supplemental oxygen should be recommended. Increased $\mathrm{FiO} 2$ application with oxygen mask or nasal cannula and High Flow Nasal Cannula (HFNC) may correct hypoxemia in these patients. In adults with COVID-19 and acute hypoxemic respiratory failure on oxygen, CCM recommended ${ }^{2}$ that $\mathrm{Spo}_{2}$ be maintained no higher than $96 \%$. The helmet mask is used to treat acute hypoxemic respiratory failure in the patients with COVID-19 without invasive mechanically ventilation. ${ }^{3}$

\section{Respiratory treatment}

Critical and most of the severe patients with COVID-19 needed respiratory treatment because of respiratory failure. For adults with COVID-19 and acute hypoxemic respiratory failure despite conventional oxygen therapy, high-flow nasal cannula (HFNC), continuous positive airway pressure (CPAP), noninvasive ventilation (NIV) or invasive mechanical ventilation (MV) should be suggested. Gattinoni and colleagues ${ }^{4}$ reported that there was two primary 'phenotypes' such Type L (characterized by Low elastance, Low ventilation-to-perfusion ratio, Low lung weight and Low recruitability) and Type $\mathrm{H}$ (characterized by High elastance, high right-to-left shunt, High lung weight and High recruitability) for the patients with COVID-19. They reported that the respiratory treatment offered to Type $\mathrm{L}$ and Type $\mathrm{H}$ patients must be different. The Type $\mathrm{L}$ patients, if hypercapnic, once intubated and deeply sedated, can be ventilated with volumes greater than $6 \mathrm{~mL} / \mathrm{kg}$ (up to $8-9 \mathrm{~mL} / \mathrm{kg}$ ) and PEEP should be reduced to $8-9 \mathrm{cmH}_{2} \mathrm{O}$, as the high compliance. Type $\mathrm{H}$ patients should be treated as severe ARDS, including higher PEEP, if compatible with hemodynamics, prone positing and extracorporeal support. However these types can be seen in the same patient depending on the course of the disease.

It was reported that barotrauma in mechanically ventilated patients with COVID-19 has been increasing. In addition, some factors such as ARDS, pulmonary embolism, pulmonary infarctions, the necrosis of pneumocytes and pulmonary fibrosis may facilitate the development of barotrauma with pulmonary parenchymal damage in the COVID-19 patients. And, it was suggested that in order to reduce the risk of barotrauma in mechanically ventilated COVID-19 patients, peak inspiratory pressure, positive end-expiratory pressure, tidal volume, plateau pressure and driving pressure slightly lower than our current practice may be recommended. ${ }^{5}$

\section{Anticoagulant treatment}

The dysfunction of endothelial cells induced by infection results in excess thrombin generation and fibrinolysis shutdown, which indicated a hypercoagulable state in patient with infection, ${ }^{6}$ such as COVID-19. It has been described in a recent study, ${ }^{7}$ severe COVID-19 is commonly complicated with coagulopathy, venous thromboembolism, and disseminated intravascular coagulation may exist in the majority of death. Tang and colleagues ${ }^{8}$ have recently reported that anticoagulant treatment is associated with decreased mortality in severe COVID-19 patients with coagulopathy. And they reported that anticoagulant therapy mainly with low molecular weight heparin to be associated with better prognosis.

\section{Anti-inflammatory drugs}

In the inflammatory phase of COVID-19, many laboratory parameters may increase. It was reported that higher level of inflammatory markers including CRP, D-dimer, ferritin and IL-6, and troponin that reflects myocardial injury were associated with higher mortality in the patients with COVID-19 in ICU followup. ${ }^{9}$ Some anti-inflammatory drugs such as corticosteroids ${ }^{10-12}$ for example dexamethasone and methylprednisolone; tocilizumab, an IL-6 receptor blockade; anakinra, ${ }^{14}$ a recombinant monoclonal antibody which is IL-1receptor antagonist; ruxolitinib, ${ }^{15}$ a selective inhibitor of Janus kinases (JAK) 1 and 2, were recommended to the treatment of the patients with COVID-19. Steroids may regulate cytokine's damaging effects by limiting the formation of cytokine, and stop macrophages and natural killer cells. In the patients with COVID-19, T-lymphocyte and macrophages produce IL-6 and cause the cytokine storm and severe inflammatory responses in lungs and multiple organs. Tocilizumab has the highest binding affinity to IL-6 receptor and lessens the inflammatory responses. However the results of these drug related to the treatment of the patients with COVID-19 are controversial.

\section{Hydroxychloroquine and combinations therapies}

Chloroquine and its derivate, hydroxychloroquine, are very closely related and used to treat malaria and rheumatologic conditions such rheumatoid arthritis, systemic lupus erythematous, and juvenile idiopathic arthritis. Yao X and colleagues ${ }^{16}$ reported that the antimalarial drugs hydroxychloroquine and chloroquine had in vitro antiviral activity and they optimized the dosage of hydroxychloroquine for the treatment of severe acute respiratory syndrome coronavirus 2 (SARSCoV-2). It was reported that the hydroxychloroquine and chloroquine could diminish various coronavirus activations such as reducing the symptom duration, exacerbation of pneumonia, including radiological improvement, and promote virus negative seroconversion. ${ }^{17}$ Because of the COVID-19 pandemic, the FDA was approved an emergency authorization for use of hydroxychloroquine and chloroquine in 2020.

In a retrospective multicenter cohort study, Rosenberg and colleagues $^{18}$ recently reported that the probability of death for patients receiving hydroxychloroquine + azithromycin was $25.7 \%$, hydroxychloroquine alone was $19.9 \%$, azithromycin alone was $10.0 \%$, and neither drug was $12.7 \%$ in patients with COVID-19 in New York State. As for adverse effect, cardiac arrest, abnormal ECG that combines arrhythmia and QT prolongation, arrhythmia overall and among ECG screened and QT prolongation among ECG screened were significantly higher in the Hydroxychloroquine + Azithromycin Group than Neither Drug Group. They concluded that treatment with hydroxychloroquine, azithromycin, or both, compared with neither treatment, was not significant associated with differences in in-hospital mortality. This is a retrospective multicenter cohort study is very interesting and we would like to write the following observations. When some data about the patient characteristic were examined in detail, we realized that the data of Hydroxychloroquine + Azithromycin Group were worse than the data of Neither Drug Group. For example; obesity (BMI $\geq 30$ ), any chronic lung conditions, respiratory rate $>22 / \mathrm{min}$, oxygen saturation $\%$, AST and ALT values $>40 \mathrm{U} / \mathrm{L}$ and abnormal chest imaging findings were worse in Hydroxychloroquine + Azithromycin Group than Neither Drug Group $(p<0.01)$. These conditions are related to higher mortality. One can associate the cause of higher mortality in the Hydroxychloroquine + Azithromycin Group with higher comorbid conditions in this group.

Adverse events reported during hospitalization in Rosenberg and colleagues $^{18}$ study haven been showed in a Table. Cardiac arrest, abnormal ECG that combines arrhythmia and QT prolongation, arrhythmia overall and among ECG screened and 
QT prolongation among ECG screened were significantly higher in the Hydroxychloroquine + Azithromycin Group than Neither Drug Group $(\mathrm{p}<0.05)$. Contrary to expectations, cardiac arrest as a cause of death was relatively lower in the Hydroxychloroquine + Azithromycin Group than Neither Drug Group, 29.7\% versus $35 \%$. The most common cause of death was respiratory failure in the both groups. Tang and colleagues ${ }^{8}$ have recently reported that anticoagulant treatment is associated with decreased mortality in severe COVID-19 patients with coagulopathy. And they reported that anticoagulant therapy mainly with low molecular weight heparin to be associated with better prognosis. Rosenberg and colleagues ${ }^{18}$ should be discussed the anticoagulant treatment and causes of death in their study. However the effects of hydroxychloroquine and combinations therapies on the patients with COVID-19 are controversial.

\section{Antiviral drugs}

Some antiviral drugs such Remdesivir, Favipiravir, Lopinavir/ Ritonavir have been recommended for treatment of COVID-19. ${ }^{19}$

Remdesivir is an antiviral drug that inhibits viral RNA polymerases. Remdesivir has been previously used for Ebola treatment than it has been recently showed its anti-coronavirus activity in vitro. ${ }^{20}$ Grein $\mathrm{J}$ and colleagues ${ }^{21}$ have reported that compassionate use of remdesivir for patients with severe Covid-19.

Favipiravir is an antiviral drug that inhibits the RNA-dependent RNA polymerase of RNA viruses. It has been reported that to be effective in the treatment of a wide range of RNA viruses. Therefore, favipiravir is considered as one of the potential antiCOVID-19 drugs according to some in vitro and clinical studies. ${ }^{22,23}$ To recommend favipiravir for the treatment of the patients with COVID-19, however, more clinical studies are needed.

Lopinavir is a protease inhibitor used to treat HIV infection, with ritonavir as a booster. These combinational drugs have used in a randomized study for the patients with severe COVID-19 but the study drugs did not decrease the time to clinical improvement compared with standard care alone..$^{24}$ The use of these antiviral drugs in the treatment of COVID-19 is still a matter of debate.

\section{Convalescent plasma therapy}

The Convalescent Plasma (CP) therapy is a kind of passive immunization like hyper immune immunoglobulin therapy for the patients with COVID-19. Generally, where a patient recovered from a viral infection with high neutralizing antibody titer against the virus would be used as plasma donors to treat the infected persons. The $\mathrm{CP}$ therapy has been used to treat several infection diseases including Spanish flu, the Ebola epidemic, influenza A H1N1 and H5N1. It has been recently reported that convalescent plasma is a potential therapy for COVID-19 in a correspondence. ${ }^{25}$ In a systemic review recently has been reported that there is very low-quality evidence to suggest efficacy and safety of CP in patients with Covid-19 infection. However, well-designed randomized trials are urgently needed to provide robust data about the effect of CP on the patients with COVID-19. ${ }^{26}$

\section{Mesenchymal stem cells therapy}

The use of mesenchymal stem cells for the treatment of patients with severe COVID-19 can be recommended. Leng $\mathrm{Z}$ et al. ${ }^{27}$ reported that the intravenously infusion of mesenchymal stem cells improved the outcome of COVID-2019 patients may be due to regulating inflammatory response and promoting tissue repair and regeneration. In a randomized controlled trial recently has been reported that the application of infusion umbilical cord mesenchymal stromal cells significantly decreased interleukin 6 in the patients with COVID-19 and increases the survival rate by modulating the immune system toward an anti-inflammatory state. ${ }^{28}$

\section{Vaccination}

Applications of various vaccines developed against corona virus still continue all over the world. It has been recently published that some adverse allergic reactions secondary to the administration of the vaccines developed for the protection of COVID-19. As a results of analyses provided by the CDC and FDA in the USA have estimated the rates of anaphylactic reactions in 2.5 and 11.1 per million of mRNA-1273 and BNT162b2 vaccines administered, respectively. ${ }^{29}$ Vaccination comes first along with protective measures to get rid of the whole world from the corona virus.

\section{Discussion}

The course of COVID-19 can be evaluated in 3 stages in general. The first stage can be defined as the viral period in which the symptoms begin, the PCR test is positive and the radiologic findings can be seen in the lung tomography. In this period, care in the service is usually sufficient. The second stage can be defined as the hyper-inflammatory period in which the clinic deteriorates, lung tomography findings progress and inflammatory parameters such as CRP, d-Dimer and ferritin increase in laboratory evaluation. During this period, advanced drug and organ support treatments, including mechanical ventilation in the intensive care unit, may be required. The third stage can be defined as the late period in which organ problems such as secondary infections and fibrosis may occur if the patient survives. These stages usually last a few weeks. However, in some variant cases, longer times and differences in laboratory values were observed such as no increase in ferritin.

Which treatment or when the therapeutic option is administered may vary depending on the severity and stage of the patient's disease. In the light of scientific studies, the clinicians should suggest the most appropriate option, according to the severity and stage of the disease, taking into account their own experience.

\section{Conclusion}

As a conclusion, since there is no specific treatment, coronavirus disease 2019 is a global problem up to now. The therapeutic options mentioned in this review should be suggested according to the severity and stage of the disease.

\section{Conflicts of interest}

The authors declare that there is no conflict of interest regarding the publication of this paper.

\section{References}

1. Huang C, Wang Y, Li X, et al. Clinical features of patients infected with 2019 novel coronavirus in Wuhan, China. Lancet. 2020;395(10223):497506.

2. Alhazzani W, Møller MH, Arabi YM, et al. Surviving Sepsis Campaign: Guidelines on the Management of Critically Ill Adults with Coronavirus Disease 2019 (COVID-19). Intensive Care Med. 2020;46(5):854-887.

3. Eroglu A, Kartal S, Saral OB. Helmet mask and tocilizumab for a patient with hemophagoctic lymphohistiocytosis syndrome and COVID-19: a case report. Brazilian Journal of Anesthesiology. 2021;71:79-83.

4. Gattinoni L, Chiumello D, Caironi P, et al. COVID-19 pneumonia:different respiratory treatments for different phenotypes. Intensive Care Medicine. 2020;46(6):1099-1102. 
5. Eroglu A. Barotrauma in mechanically ventilated patients with COVID-19. Minerva Anesthesiol. 2021;87(2):144-146.

6. Levi M, van der Poll T. Coagulation and sepsis. Tromb Res. 2017; 149:38-44.

7. Tang N, Li D, Wang X, et al. Abnormal coagulation parameters are associated with poor prognosis in patients with novel coronavirus pneumonia. J Thromb Haemost. 2020.18(4):844-847.

8. Tang N, Bai H, Chen X, et al. Anticoagulant treatment is associated with decreased mortality in severe coronavirus disease 2019 patients with coagulopathy. J Thromb Haemost. 2020;18(5):1094-1099.

9. Kartal S, Kongur E, Eroglu A. A research of the factors associated with mortality of the patients with COVID-19 in the intensive care unit. Anesthesia \& Pain Research. 2021;5(1):1-5.

10. Wu C, Chen X, Cai Y, et al. Risk factors associated with acute respiratory distress syndrome and death in patients with coronavirus disease 2019 pneumonia in Wuhan, China. JAMA Intern Med. 2020;180(7):934-943.

11. Yang Z, Liu J, Zhou Y, et al. The effect of corticosteroid treatment on patients with coronavirus infection: a systematic review and metaanalysis. J Infect. 2020;81(1):e13-e20.

12. Comparon $\mathrm{C}$, Boubaya $\mathrm{M}$, Sritharan $\mathrm{N}$ et al. A short course of corticosteroids reduces the risk of mechanical ventilation and death in patients with moderate to severe COVID 19 pneumonia: results of a retrospective monocentric cohort. Infect Dis (Lond). 2021;1-10.

13. Zhao J, Cui W, Tian B. Efficacy of tocilizumab treatment in severely ill COVID-19 patients. Crit Care. 2020;24(1):1-4.

14. Barkas F, Ntekouan SF, Kosmidou M, et al. Anakinra in hospitalized nonintubated patients with coronavirus disease 2019: a systematic review and meta-analysis. Rheumatology (Oxford). 2021:keab447.

15. Richardson P, Griffin I, Tucker C, et al. Baricitinib as potential treatment for 2019-nCoV acute respiratory disease. Lancet. 2020;395:e30-e31.

16. Yao $\mathrm{X}$, Ye F, Zhang $\mathrm{M}$, et al. In vitro antiviral activity and projection of optimized dosing design of hydroxychloroquine for the treatment of Severe Acute Respiratory Syndrome Coronavirus 2 (SARS-CoV-2). Clin Infect Dis. 2020. 71(15):732-739.
17. Gao J, Tian Z, Yang X. Breakthrough: chloroquine phosphate has shown apparent efficacy in treatment of COVID-19 associated pneumonia in clinical studies. Biosci Trends. 2020;14(1):72-73.

18. Rosenberg ES, Dufort EM, Udo T, et al. Association of treatment with hydroxychloroquine or azithromycin with in-hospital mortality in patients with COVID-19 in New York State. JAMA. 2020;323(24):2493-2502.

19. Yavuz SS, Unal S. Antiviral treatment of COVID-19. Turk J Med Sci. 2020;50:611-619.

20. Wang M, Cao R, Zhang L, et al. Remdesivir and chloroquine effectively inhibit the recently emerged novel coronavirus $(2019-\mathrm{nCoV})$ in vitro. Cell Research. 2020;30:269-271.

21. Grein J, Ohmagari N, Shin D, et al. compassionate use of remdesivir for patients with severe Covid-19. New England Journal of Medicine. 2020.

22. Du YX, Chen XP. Favipiravir: pharmacokinetics and concerns about clinical trials for 2019-nCoV infection. Clin Pharmacol Ther. 2020;108(2):242-247.

23. Xinhua Net. Favipiravir shows good clinical efficacy in treatingCOVID-19: official.

24. Cao B, Wang Y, Wen D, et al. A trial of lopinivar/ritonavir in adults hospitalized with severe Covid-19. New England journal of Medicine. 2020 .

25. Chen L, Xiong J, Bao L. Convalescent plasma as a potential therapy for COVID-19. Lancet Infect Dis. 2020;20:398-400.

26. Seth T, Elavarasi A, Sahoo RK, et al. Convalescent plasma therapy for Covid-19: A systematic review. Natl Med J India. 2020;33(4):213-221.

27. Leng Z. MSC transplantation improves COVID-19 patient outcome. Aging Dis. 11; 2020.

28. Dilogo IH, Aditianingsih D, Sugiarto A, et al. Umbilical cord mesenchymal stromal cells as critical COVID-19 adjuvant therapy: A randomized controlled trial. Stem Cells Transl Med. 2021.

29. Cabanillas B, Novak N. Allergy to COVID-19 vaccines: A current update. Allergol Int. 2021;S1323-8930(21)00045-00049. 